\title{
VELOCITY AND TOTAL PRESSURE MEASUREMENTS IN THE TWO-STAGE HYBRID THRUSTER TIHTUS
}

\section{H. Böhrk and M. Auweter-Kurtz}

\begin{abstract}
A novel two-stage thermal-inductive hybrid thruster of the Universität Stuttgart (TIHTUS) is under development at the Institut für Raumfahrtsysteme (IRS). It provides the possibility to supply power and mass-flow through either stage into the plasma. A variation of power- and massflow-staging was investigated with a Pitot probe and an electric timeof-flight probe. The present paper discloses the radially resolved measurement results of total pressure and plasma velocity at a downstream position of $200 \mathrm{~mm}$. Furthermore, a method is presented to access radially resolved flow temperature from the combined pressure and velocity measurement. The temperature data are also presented. For a sum of powers of $50 \mathrm{~kW}$ at $300 \mathrm{mg} / \mathrm{s}$, the Pitot pressure maximum is at $85 \mathrm{~Pa}$ while maximum velocity is measured as 9,224 and $10,350 \mathrm{~m} / \mathrm{s}$ for two respective conditions. On the plume axis, the temperatures reach up to $9000 \mathrm{~K}$.
\end{abstract}

\section{THRUSTER CONCEPT}

The current limitations to the exhaust velocity of hydrogen previoulsy heated in the plenum of a rocket engine are imposed due to various reasons. At low stagnation pressures, the high bulk enthalpy added, although high, is largely transformed into dissociation and ionization but cannot be directed to kinetic energy when expanded to lower pressures in a nozzle. Or the bulk enthalpy is, although useful, low at high stagnation pressures. Or, finally, the enthalpy that, at optimum pressure, can be converted to directed kinetic energy, shows a maximum but is a compromise between the two features mentioned above [1]. When both, gas temperature and stagnation pressure, are increased, the optimum will collide with limitations due to the electrode material. Therefore, new thruster concepts are required in order to produce relatively high exhaust velocity at relatively high thrust. 


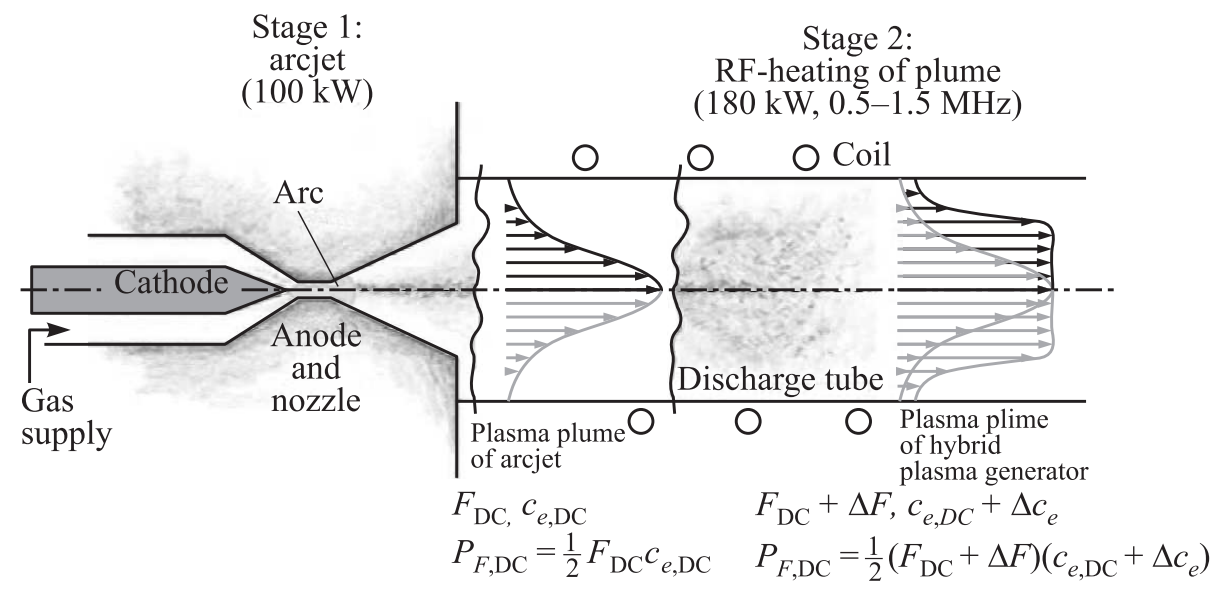

Figure 1 Principle of TIHTUS

Exhaust velocity $c_{e}$ of any thermal thruster is dependent on the propellant temperature $T_{0}$ in the combustion chamber and its molecular mass $M$ according to

$$
c_{e} \propto \sqrt{\frac{T_{0}}{M}} .
$$

The average propellant temperature should, therefore, be as high as possible. Currently, the highest exhaust velocities of thermal propulsion concepts that use stationarily chamber-heated hydrogen range between 20 and $25 \mathrm{~km} / \mathrm{s}[2]$.

Arcjet thrusters have been developed throughout the past decades. They provide relatively high thrust at moderate exhaust velocities. An arcjet thruster has a central cathode and an annular anode. The propellant is injected into the ring-shaped gap between the two electrodes and heated up by an electric arc. It is then expanded and accelerated through a nozzle where the energy contained in the plasma is transferred to directed energy. The plasma flow of a thermal arcjet is characterized by high specific enthalpy and high flow velocity. These characteristics are, however, combined with the presence of steep radial property gradients as in a hot, energy-rich core with a relatively cold gas layer at its edge.

An idea to transfer more power into the plasma flow is to reheat these edges of the plume by a second heating mechanism in the way of an afterburner. The principle is sketched in Fig. 1.

TIHTUS is a two-stage plasma generator where reheating is realized by means of inductive heating. This new concept has the potential of producing large thrust and high effective exhaust velocity by addition of power in the second stage $[3,4]$. It is therefore considered the predecessor of a future 
Table 1 Nomenclature for operational conditions

\begin{tabular}{lcccc}
\hline & $\begin{array}{c}P_{\mathrm{DC}}, \\
\mathrm{kW}\end{array}$ & $\begin{array}{c}P_{\mathrm{RF}}, \\
\mathrm{kW}\end{array}$ & $\begin{array}{c}\dot{m}_{\mathrm{DC}}, \\
\mathrm{mg} / \mathrm{s}\end{array}$ & $\begin{array}{c}\dot{m}_{\mathrm{RF}}, \\
\mathrm{mg} / \mathrm{s}\end{array}$ \\
\hline $\mathrm{T} 50|00-300| 0$ & 50 & 0 & 300 & 0 \\
$\mathrm{~T} 25|25-300| 0$ & 25 & 25 & 300 & 0 \\
$\mathrm{~T} 20|30-300| 0$ & 20 & 30 & 300 & 0 \\
\hline $\mathrm{T} 25|25-200| 100$ & 25 & 25 & 200 & 100 \\
$\mathrm{~T} 25|25-100| 200$ & 25 & 25 & 100 & 200 \\
\hline
\end{tabular}

propulsion system for the transport of heavy payload on interplanetary trajectories $[5,6]$.

TIHTUS consists of an arcjet thruster (first stage) and an inductively heating afterburner (second stage). The second stage consists of a discharge tube and a coil spun around it, being a part of a resonance circuit. The inductively heated plasma generator (IPG) is used as the afterburner since in induction heating, due to the skin-effect, the power is coupled into the plasma at near-coil position, where the cold gas layer of the arcjet plume is located [7]. The plasma jet emerging from the arcjet is thus expanded into the quartz discharge tube. The alternating radio-frequency $(\mathrm{RF})$ current in the coil induces an oscillating, mostly azimuthal, magnetic field inside the tube. In turn, this field initiates an electric discharge in the propellant oriented in the opposite direction of the coil current. The free electrons contained in the plasma from the thermal arcjet are accelerated by the electric field and by means of collisions they transfer their induced power to atoms and molecules. Radio-frequency power is thus coupled into the plasma.

It has been shown that with the currently used setup, continuous two-stage operation of the thruster with hydrogen as propellant is possible [3]. The principal question is whether it is possible to specifically heat the outer edges of an arcjet plume so that higher exhaust velocity can be attained. Of course, a dependency of power to each stage is expected as well as a dependency of the gas mass flow rate is evident.

The thruster is operated as a water-cooled model using hydrogen as propellant. Power may be coupled into the arc heated (DC) or the inductively heated (RF) stage. Gas flow through the arc heated stage is expanded first into the injection head of the inductive, second, stage where a swirl gas flow can be admixed. The operational conditions are therefore declared as $\mathrm{T} P_{\mathrm{DC}} \mid P_{\mathrm{RF}^{-}}$ $\dot{m}_{\mathrm{DC}} \mid \dot{m}_{\mathrm{RF}}$ throughout this paper. The investigated conditions are listed in Table 1 .

The radial profiles of total pressure and plasma flow velocity are measured by means of a Pitot probe and a time-of-flight electric probe, respectively. As mentioned above, the propellant temperature is of main interest. However, high 
temperature is a very delicate state variable and measurements of pressure, temperature, and velocity are difficult to carry out in supersonic and low-pressure flow of plasma, especially if one wishes to obtain local values. In the present work, using the measured data of velocity by electric time-of-flight probes and total, or Pitot, pressure, a method based on an assumption of thermodynamic equilibrium will be presented to deduce temperature information. Other methods of velocity and temperature measurement exist apart from the ones used here. Especially optical measurements are ideal for these measurements but require a complex optical system. Laser induced fluorescence (LIF) and emission spectroscopy are often applied for the measurement of hydrogen temperature [810]. Laser induced fluorescence and, even more likely, Fabry Perot interferometry are suitable means to measure velocity $[8,9,11,12]$, although emission is low at the plume edge. Moreover, heavy particle temperature can be determined from crossed electric single probe signals when the plasma velocity is already known $[11,13]$ and velocity can be measured by induction velometry [14].

After having introduced the thruster principle above, this paper will present the setup of the ground test facility and the setup of the Pitot and time-of-flight probes. Furthermore, the methodology is introduced, by means of which temperature information is derived from Pitot pressure and velocity. In the results section, radial profiles of total pressure and velocity are shown for the listed operating conditions and interpreted. Independent temperature measurements must be performed in order to prove the validity of the assumptions made here. These are, however, not included in the present paper.

\section{EXPERIMENTAL APPARATUS}

The TIHTUS ground test facility consists of the two plasma sources and the vacuum chamber. TIHTUS first stage is HIPARC-W(water-cooled) and foreseen for up to 100-kilowatt operation. It is supplied by a 6-megawatt-DC-power supply. The second stage is an IRS-IPG configuration with 3.5 coil windings and four capacitors, resulting in a nominal operational frequency of $840 \mathrm{kHz}$. The device is connected to a 180-kilowatt RF power supply able to be operated at frequencies ranging from 0.5 to $1.5 \mathrm{MHz}$. The scheme of the facility is depicted in Fig. 2. Installed are a gas supply system, water-cooling system and a data acquisition system. The size of the vacuum chamber is about $2.5 \mathrm{~m}$ in length and $2 \mathrm{~m}$ in diameter. Its forward end cap carries the thruster and the external resonance circuit. The rear end of the chamber is connected to the IRS vacuum pump system, the total suction power of which amounts to $6000 \mathrm{~m}^{3} / \mathrm{h}$ at atmospheric pressure or $250000 \mathrm{~m}^{3} / \mathrm{h}$ at $10 \mathrm{~Pa}$.

Both parts of the thruster are water-cooled at present. Therefore, coolant flow rates are measured as are the flow rates of the propellant. However, at a 


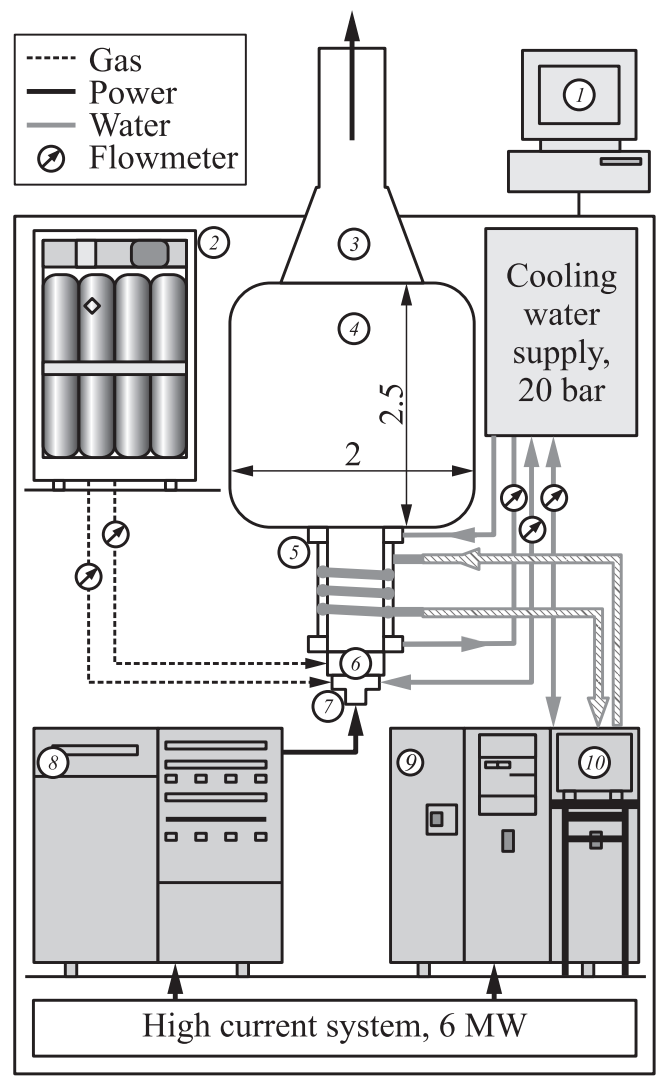

Figure 2 Scheme of the experimental apparatus: 1 - data acquisition system; $2-$ gas supply; 3 - pump system $\left(6000 \mathrm{~m}^{3} / \mathrm{h}\right.$ at $\left.1 \mathrm{bar}\right) ; 4$ - vacuum tank (probes and optical access); 5 - inductively heated plasma generator; 6 - gas injection for swirl gas; 7 - arcjet thruster; 8 - DC generator; 9 - high-frequency generator; and $10-$ capacitors (resonance circuit 1-4 MHz). Dimensions are in meters

further stage of development, the strategy foresees building the plasma source in a radiation-cooled design, which promises another gain in specific impulse.

For plume investigation, a two-axes table is installed inside the vacuum tank on which probes can be mounted.

\subsection{Pitot Probe}

The total, or Pitot, pressure is the pressure present in the foremost stagnation point of a body inserted into a plasma column, such as a Pitot probe. Within 


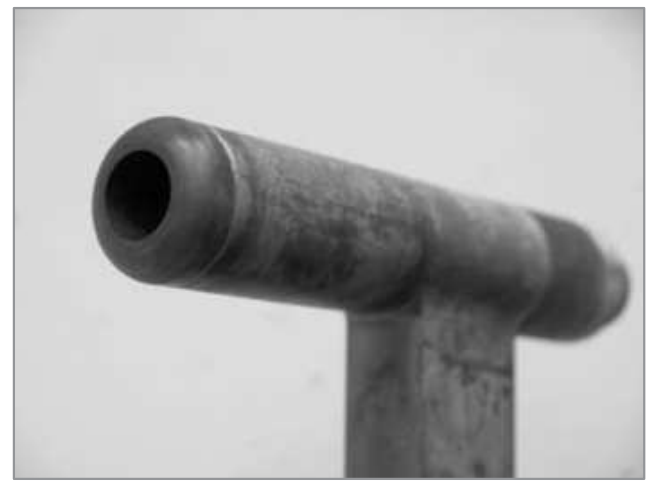

Figure 3 Pitot probe

the probe, the deceleration of the flow takes place isentropically. The pressure measured at the end of a Pitot tube, therefore, corresponds to the total pressure in the stagnation point in front of the tube opening. At Mach number $M \geq 1$, this is the plenum pressure just behind an imaginary vertical, ideal bow shock. In a supersonic flow, the Pitot probe, therefore, does not measure the total pressure in the free stream. The ratio of the true total pressure of the free stream $p_{\text {tot, } 1}$ to the measured total pressure behind a bow shock $p_{\text {tot,2 }}$ is [15]

$$
\frac{p_{\text {tot }, 1}}{p_{\text {tot }, 2}}=\left(\frac{2 \kappa}{\kappa+1} \mathrm{M}^{2}-\frac{\kappa-1}{\kappa+1}\right)^{1 /(\kappa-1)}\left(\frac{1+((\kappa-1) / 2) \mathrm{M}^{2}}{((\kappa+1) / 2) \mathrm{M}^{2}}\right)^{\kappa /(\kappa-1)}
$$

The Pitot probe used in the present case is of European standard geometry (flat nose, 50-millimeter body diameter, rounded edge) with a measurement orifice of 26.5-millimeter diameter. It is water-cooled, connected to ground potential and depicted in Fig. 3. Both probe cooling and ohmic heating of the probe by electromagnetic effects affect the Pitot pressure measurement. However, earlier works have shown that the two effects counteract each other and stay below $6.4 \%$, whereas the probe cooling's effect is between twice and four times as high as the electromagnetic effect [16].

Furthermore, the measured total pressure is considered not to be influenced by viscosity effects or axial pressure gradients. The mean free path of the charged gas at $<1 \mathrm{hPa}$ is on the order of a millimeter. Compared to the Pitot tube diameter, this results in a Knudsen number of $\mathrm{Kn} \approx 0.4$, while compared to the Pitot probe outer diameter, it is $\mathrm{Kn} \approx 0.2$. For proof of reproducibility, the measurement points were detected twice and averaged values are presented here. 


\subsection{Electric Time-of-Flight Probe}

For measuring the plasma velocity $v_{\infty}$, electric time-of-flight probes are applied. Electric probes are electrically conductive measurement devices exposed to a plasma. A set of two floating double probes are used, so that the plasma's RFtime-variant potential is not detected and mixed in with the measurement signal. A double probe has two electrodes with a voltage applied between them. Due to an electrically conductive plasma passing between the electrodes, a current can be measured. The measurement principle for plasma velocity is based on the axial offset of two double probes. The current flow between either pair shows fluctuations in the plasma which translate with plasma velocity from the first to the second double probe, i.e., a defined distance [17]. The principle is depicted in Fig. 4. The electrodes are of tungsten wire with a diameter of $0.5 \mathrm{~mm}$ and the two double probes have an axial offset of $26 \mathrm{~mm}$. An example of the probe signals are shown in Fig. 5 and it can be seen that the fluctuation moves from one double probe to the next one in $\Delta t=3.4 \mu \mathrm{s}$ and so do the particles [17]. At each radial position five pairs of curves were recorded. At ten occurences,

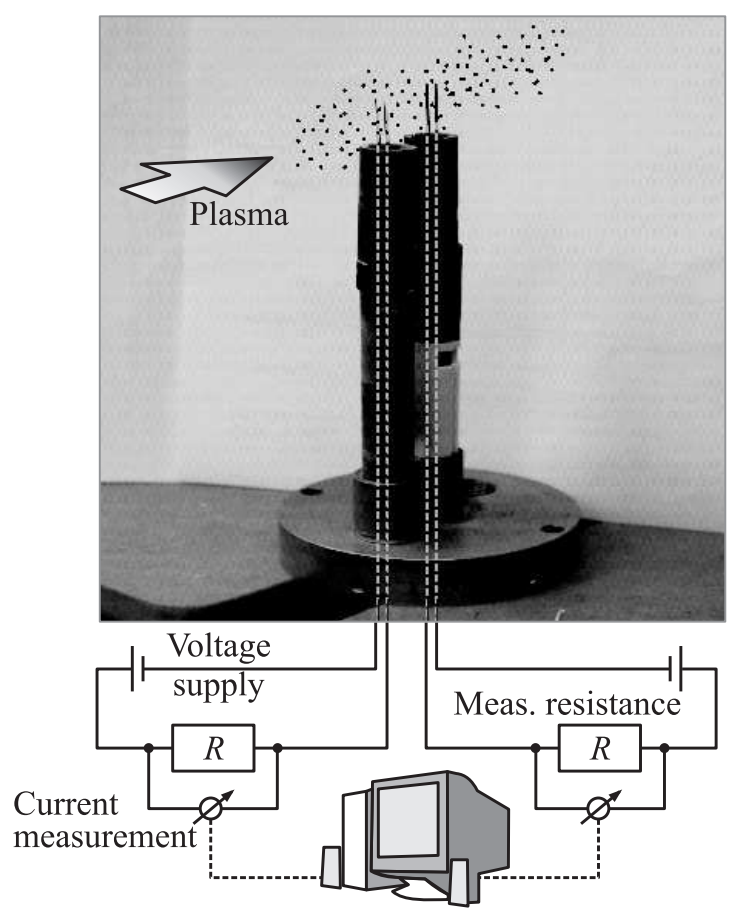

Figure 4 Electric time-of-flight probe setup 


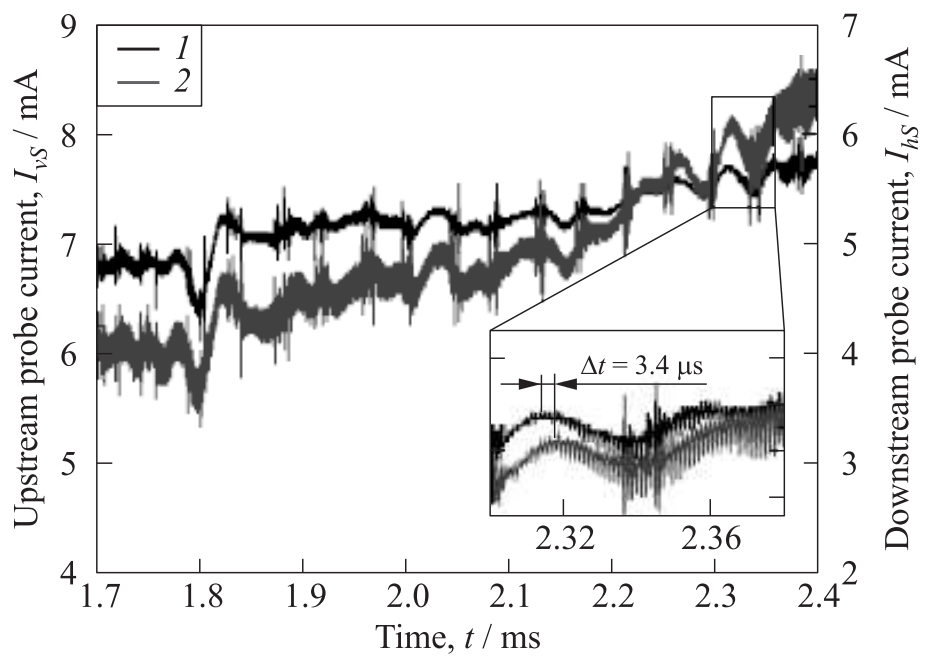

Figure 5 Probe data from operational condition T 25|25-100|200

the time delay of the measurement was averaged for each of the five signals. The velocities determined from their correlation according to $v=(0.026 \mathrm{~m}) / \Delta t$ were then averaged. For pure DC-plasma, this procedure can be done by a computational cross-correlation. However, for the RF plasma that reflects the operating frequency of the inductively heated stage, the results of computational cross-correlation were highly erratic. The data analysis was therefore conducted by hand.

Although the time-variant plasma potential had been compensated for by chosing double probes, voltage is induced into the electrodes by the RF magnetic field. It is dependent upon the magnetic field strength $B$ and the projected area $A_{\text {proj }}$ of the electrodes according to

$$
V_{\text {ind }}=\dot{B} A_{\text {proj }}+B \dot{A}_{\text {proj }}
$$

Consequently, the probes are arranged so that all four cylindrical probes of the two double probes are axially aligned with the plume axis so that the projected area is minimized.

\section{METHODOLOGY}

In the present elaboration, the plume of two-staged TIHTUS thruster is investigated by a Pitot probe and an electric time-of-flight probe. A novel method 
is introduced here, in which from total pressure and velocity, temperature is deduced. The method is based on an assumption of thermodynamic equilibrium. In order to prove the validity of the assumptions made, independant temperature measurements must be performed. These could, for example, be done by emission or absorption spectroscopy or, since velocity is already known, by crossed electric single probes.

Mach number is defined as

$$
\mathrm{M}=\frac{v}{a}=\frac{v}{\sqrt{\kappa R T}}
$$

with the speed of sound $a$, the ratio of specific heats $\kappa$, and the specific gas constant $R$. Depending on the Mach number, in the stagnation point a total pressure is formed so that

$$
\frac{p_{\text {tot }}}{p_{\infty}}=\left(\frac{\kappa+1}{2} \mathrm{M}^{2}\right)^{\kappa /(\kappa-1)}\left(2 \frac{\kappa}{\kappa+1} \mathrm{M}^{2}-\frac{\kappa-1}{\kappa+1}\right)^{1 /(1-\kappa)}
$$

in supersonic and

$$
\frac{p_{\text {tot }}}{p_{\infty}}=\left(1+\frac{\kappa-1}{2} \mathrm{M}^{2}\right)^{\kappa /(\kappa-1)}
$$

in subsonic flow [15]. The ratio of specific heats $\kappa$ is a function of temperature. It was shown by Laure [18] and Hiers and Pruitt [19] that a deviation has only little influence on the result of Mach number from Eq. (3). For the range of $1.1<\kappa<1.67$, it is found that the Mach number resulting from Eqs. (2) and (3) deviated at maximum of $15 \%$ in the expected range between $0.5<\mathrm{M}<3$. It was shown by Krause that the cooling of the flow by the water-cooled Pitot probe had only little effect on the pressure signal [16]. The data presented here are furthermore based on the assumption that the ambient pressure in the vacuum tank is imposed on the plasma plume. This assumption is also supported by Refs. [20, 21].

Just like the ratio of specific heats $\kappa$, the specific gas constant $R$ is dependent of temperature. In the present study, their values were taken from [22] and are based on a plasma composition model assuming thermodynamic equilibrium. However, whether the present low-density plasma is in chemical and thermal equilibrium is unknown. Therefore, as an approach, equilibrium is assumed for the temperature determination which still needs to be proven by direct measurements. Transferring Eq. (2) to a function for velocity $v$ of temperature $T$ results in

$$
v^{2}=\mathrm{M}^{2} \kappa(T) R(T) T .
$$

The solid curve in Fig. 6 shows the plasma velocity in the plume center as derived from Eq. (4) for the given temperature range and total pressure ratio measured at $\mathrm{T} 50|00-300| 0$. For the same operating point and position, the 


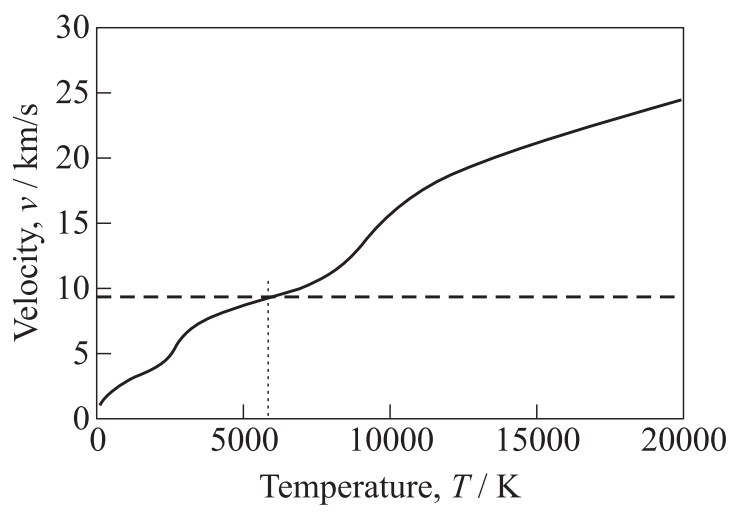

Figure 6 Scheme of temperature determination method

velocity measured by electric probes is marked in the graph as a dashed horizontal line.

In the figure, the solid velocity curve derived from Pitot probe measurements for a variety of temperatures and the dashed velocity curve measured with electric probe intersect. Thus, the temperature for which the velocity is derived from Fig. 6 is the plasma temperature at the operating position. This means that the local temperature is derived from velocity and total pressure measurements while only two assumptions have to be made. They are that of a perpendicular ideal bow shock ahead of the Pitot probe and that of plasma fluctuations (measured by the electric probe) moving with plasma velocity. Furthermore, the ratio of specific heats and specific gas constant were calculated from a model considering thermochemical equilibrium [22].

The derived plasma temperature is the local static equilibrium temperature. Since, in fact, thermodynamic equilibrium is unlikely in the rarefied flow, by means of the presented method, the heavy particle temperature is typically overestimated whereas the electron temperature is underestimated.

In the framework of the present measurement campaign, a radial profile of plasma velocity was measured for the five operating conditions of Table 1 . Hence, for each point of measurement in each radial profile, the temperature is determined from the Pitot probe and electric time-of-flight measurements.

\section{RESULTS AND INTERPRETATION}

As was described above, in TIHTUS, electric power can be applied either to the arc heated stage or to the inductively heated stage, or to both stages. Also, 
the ratio of the gas flows through either stage can be varied. Five operating conditions listed in Table 1 were investigated. They include a variation of power staging for a gas flow ratio of $300 \mid 0 \mathrm{mg} / \mathrm{s}$ and a variation of mass flow staging for constant power staging of $25 \mid 25 \mathrm{~kW}$.

The radial profiles of total pressure and plasma flow velocity are measured by means of the Pitot probe and a time-of-flight electric probe, respectively. As mentioned, propellant temperature is of the main interest and the radial temperature profile will be obtained from the method described above.

Radial profiles were investigated at an axial position of $x=200 \mathrm{~mm}$ downstream of the thruster exit.

\subsection{Pitot Pressure}

Figure $7 a$ shows the Pitot pressure in the plane perpendicular to the plume exhaust direction with a mass flow rate of $300 \mathrm{mg} / \mathrm{s}$ through the arcjet thruster stage. The maxima at eccentric position originate from the plasma flow through the discharge tube instead of a diverging nozzle. This shape was observed in other plasma jets obtained by arcjet plasma sources and corresponds to the free jet theory [20]. The curve marked with hollow triangles represents only arc heated stage active at $20 \mathrm{~kW}$. It shows maxima at eccentric position, while the plume is quite narrow. Black triangles mark the same arcjet power with the second stage power added to it. It becomes evident, that the power of the inductively heated stage is coupled into the plasma annularly at eccentric position near to the coil as predicted for the inductive stage [7]. For reasons of clarity in the figure, error bars are marked only in the curve for T 20|30-300|0. They origin from the standard deviation of the measurements plus the pressure gauge error.

The black symbols represent conditions from Table 1 with power staging adding up to $50 \mathrm{~kW}$ each. At the plume center, it can be observed that with increasing arc power, higher total pressure is produced. The highest maxima, representing a total pressure of $0.85 \mathrm{hPa}$, are achieved with $20 \mathrm{~kW}$ supplied from the arc heating stage and $30 \mathrm{~kW}$ coupled into the plume by inductive heating. The same condition shows the widest plume diameter. This can be interpreted as the most power coupled into the plasma at coil-near position. However, the smallest diameter is not reached at pure arc heating with $50 \mathrm{~kW}$ but with 25 kilowatt arc and 25-kilowatt inductive heating.

For a power staging of $25 \mid 25 \mathrm{~kW}$, a gas flow variation was conducted. The Pitot pressure graphs are presented in Fig. $7 b$. From the edges of the plume, it can be seen that, according to the measurement condition and the mass flow staging, the ambient pressure in the tank varies between 0.34 and $0.4 \mathrm{hPa}$. In the second stage, the gas flow is injected radially as a swirl gas. It is observed that the more gas is injected as a swirl gas, the higher the ambient pressure. 


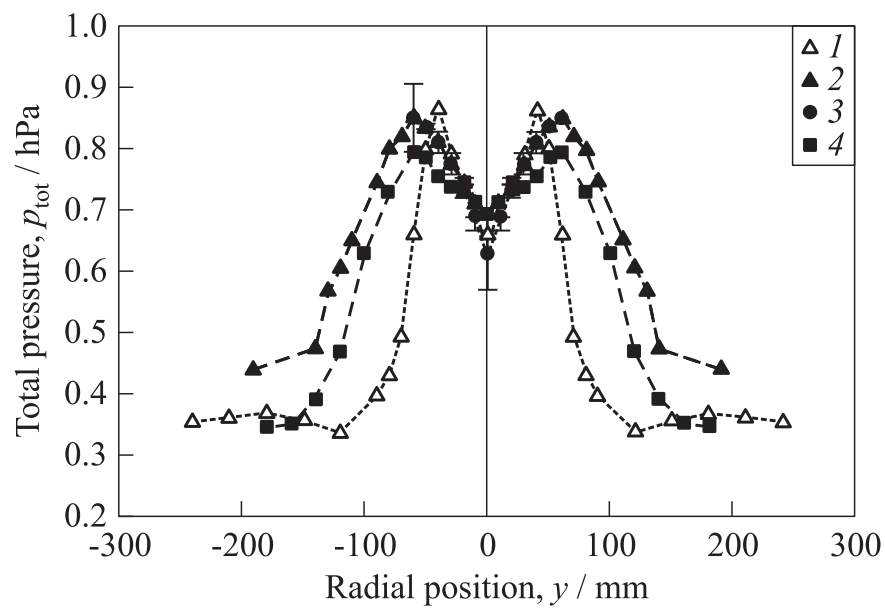

(a)

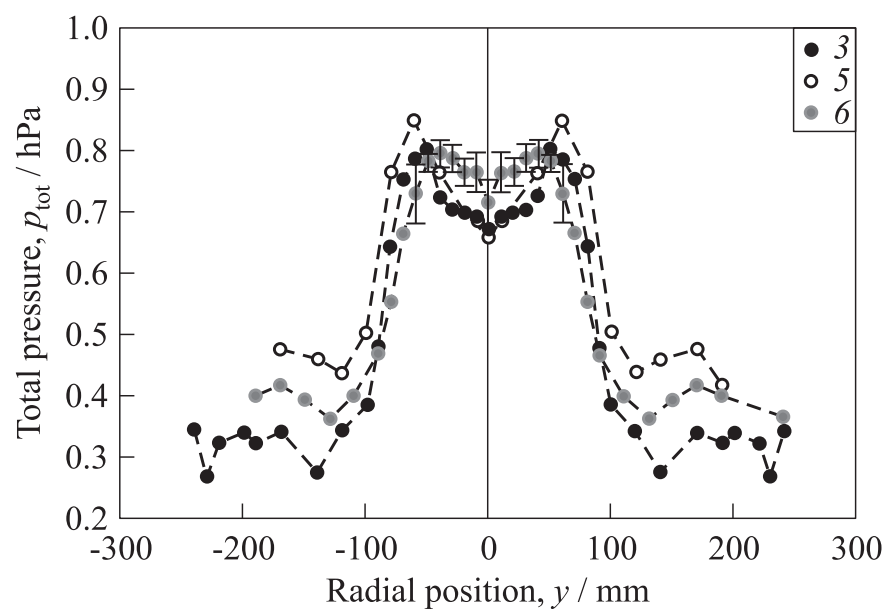

(b)

Figure 7 Pitot pressure at variation of $(a)$ power partitioning: $1-$ T 20|00-300|0; 2 - T 20|30-300|0; 3 - T 25|25-300|0; and 4 - T 50|00-300|0; and(b) mass flow rate partitioning: 5 - T 25|25-100|200; and 6 - T 25|25-200|100

The diameter of the plasma plume, however, does not remarkably change with varying mass flow staging. Total pressure maxima of $0.85 \mathrm{hPa}$ are attained for $100 \mid 200 \mathrm{mg} / \mathrm{s}$.

From these measurements, the highest integral of total pressure is reached with T 20|30-300|0. The data are compared to thrust measurements in [4]. 


\subsection{Velocity}

In Fig. 8, plasma velocities measured with the electric time-of-flight probes are depicted. Measurements could only be taken in the plume center, where ionization of the plasma was high enough to have a current flow through the plasma and the electric probes. Profiles were able to be recorded out to a radial distance of $150 \mathrm{~mm}$ from the plume center.

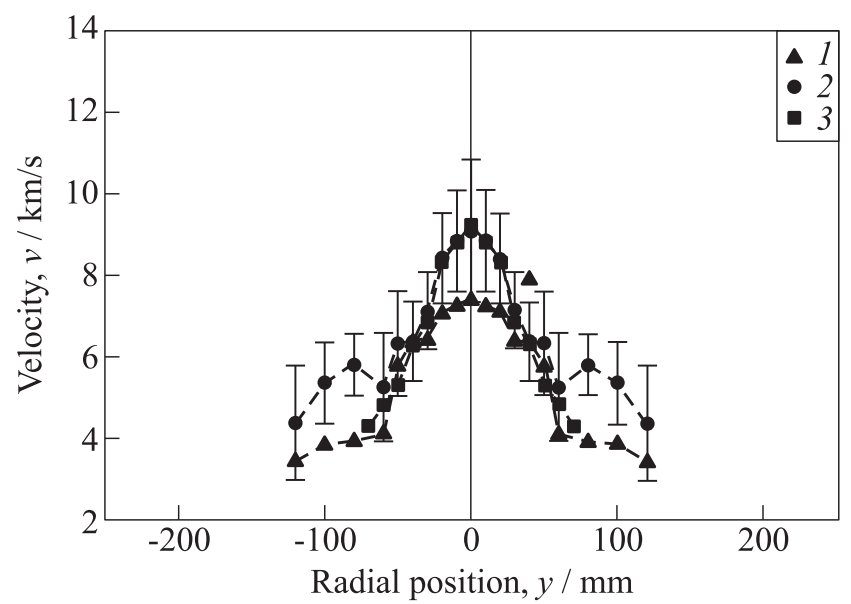

(a)

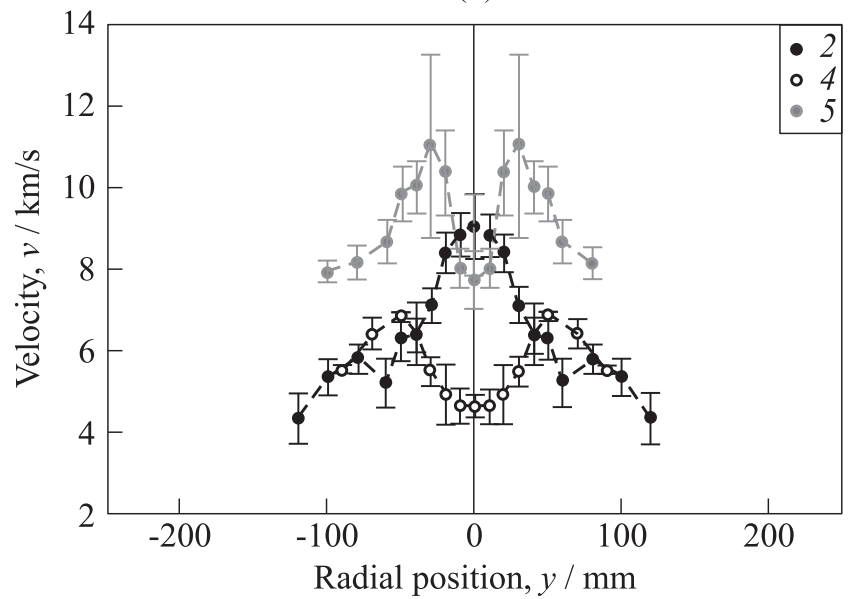

(b)

Figure 8 Velocity at variation of $(a)$ power partitioning: 1 - T 20|30-300|0; $2-$ $\mathrm{T} 25|25-300| 0$; and $3-\mathrm{T} 50|00-300| 0$; and (b) mass flow rate partitioning: $4-$ T 25|25-100|200; and 5 - T 25|25-200|100 
Figure $8 a$ represents the variation of power staging according to the pressure data presented above. The curves do not have the eccentric maximum shape as the Pitot pressure curves. They demonstrate a maximum at central position. The center values, according to the Pitot center values, increase with increasing the first stage power. Power partitioning of $25 \mid 25$ reaches almost the maximum velocity of $9224 \mathrm{~m} / \mathrm{s}$ in the plume center that were reached by the one of $50 \mid 0$ with an addition of power at the plume edge. Of much smaller amount is the maximum of the power staging with $20 \mid 30 \mathrm{~kW}$. This means that when too little power is applied in the first stage, ionization is not high enough for the induction coil to efficiently couple power into the plasma. Furthermore, velocity decreases while the profile gains in diameter.

At a maintained power ratio of $25 \mid 25 \mathrm{~kW}$, the gas flow variation in Fig. $8 b$ shows that with pure flow through the arcjet, a maximum is formed in the plume center. However, for increasing swirl gas flow, the maximum becomes eccentric. This is most extreme for $100 \mathrm{mg} / \mathrm{s}$ of hydrogen fed through the arcjet and $200 \mathrm{mg} / \mathrm{s}$ fed through the injection head. The highest local velocity of $10,350 \mathrm{~m} / \mathrm{s}$ is reached with $200 \mathrm{mg} / \mathrm{s}$ of hydrogen fed through the arcjet and $100 \mathrm{mg} / \mathrm{s}$ fed through the injection head. The plume centers show an increase in velocity with increasing arcjet flow.

\subsection{Temperature}

Using the method described above, the temperature is derived from the Pitot pressure and plasma velocity. The ratio of specific heats used was that from [22] providing data depending on temperature and static pressure. Since the temperature is derived iteratively, the error can only be determined from the reflection that according to Eq. (4), $T=v^{2} /\left(\mathrm{M}^{2} \kappa(T) R(T)\right)$, the plasma temperature is high when Mach number is small and velocity is large. From this consideration, it follows that $T+\Delta T$ for $p_{\text {tot }}-\Delta p_{\text {tot }}$ and $v+\Delta v$ and vice versa. The corresponding errors are indicated in Fig. 9.

It becomes evident from Fig. $9 a$ that for all operating conditions using pure central gas flow at $300 \mathrm{mg} / \mathrm{s}$, the temperature profile is, in pronciple, similar at any power staging with a maxiumum in the plume center. This indicates that the thermal efficiency of the coupling of the inductive, second, stage into the plasma is high. Equation (4) shows that temperature is quadratically proportional to velocity but inversely proportional to $\left(\left(p_{\text {tot }} / p_{\infty}\right)^{f(\kappa)}\right)^{2}$. The ratio of specific heats lies between 1 and 1.67 and for high total pressure, the temperature must be low while for high velocity, the temperature must be high.

Figures $7 a$ and $8 a$ show that Pitot pressure has eccentric maxima while velocity does not. Therefore, at increasing distance from the center, the temperature shows steep temperature gradients, since velocity falls and total pressure rises. 


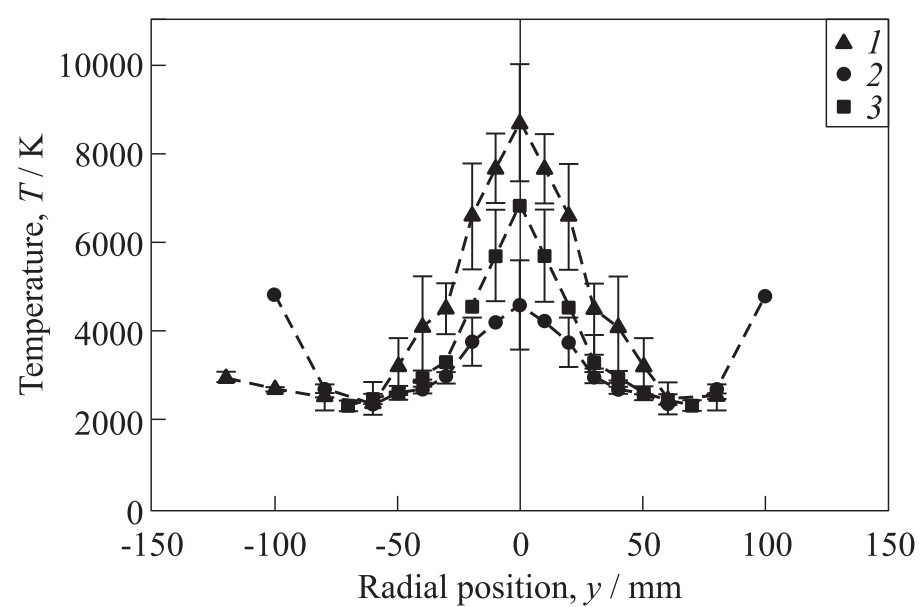

(a)

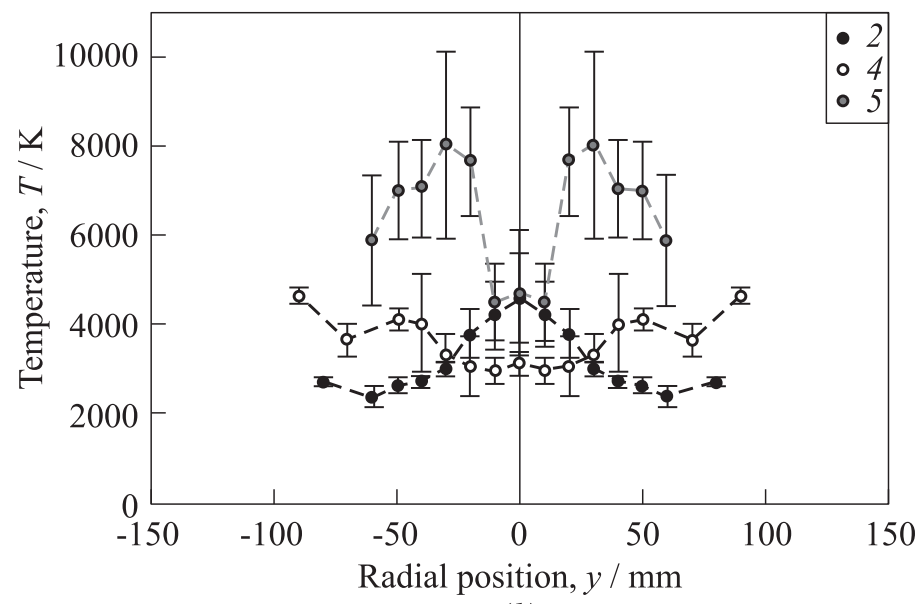

(b)

Figure 9 Temperature at variation of $(a)$ power partitioning: 1 - T 20|30-300|0; 2 - T 25|25-300|0; and 3 - T 50|00-300|0; and (b) mass flow rate partitioning: $4-$ T $25|25-100| 200$; and $5-\mathrm{T} 25|25-200| 100$

Maximum temperature is derived for T 20|30-300|0 with $8689 \mathrm{~K}$, indicating that the plasma is strongly inductively heated but the temperature cannot be used since the flow is not expanded through a nozzle unlike the consideration of Eq. (1). At T 50|00-300|0, the temperature is about $1850 \mathrm{~K}$ lower, i.e., $6841 \mathrm{~K}$. The increase at the edge of the calculated area is unphysical and occurs due to scaling problems in the computation. 
For the variation of mass flow rate staging, depicted in Fig. 9b, it is found that, according to the behavior of the velocity, with increasing swirl gas flow, the maxima become eccentric and diverge. However, with pure arc heated flow, expanded through the arcjet nozzle and next through the discharge tube, there is a temperature maximum of $4583 \mathrm{~K}$ in the plume center. It was expected for the flow to have increasing temperature in the plume center for decreasing central gas flow since a constant $25 \mathrm{~kW}$ are coupled via the arc to decreasing mass flow rate. Still, the measurements show decreasing temperature in the plume for increasing swirl gas flow. This indicates that the cold swirl gas mixes with the central gas. This is possible since by means of the discharge tube of the second stage, the plume cannot unfold as it would but is forced against the cooled injection head where, additionally, a cold gas stream is injected. This effect is also observed by thrust measurement [4].

Equation (1) had shown that maximum effective exhaust velocity can be reached with the hottest plasma. The plasma with the maximum integral temperature is generated with T $25|25-300| 0$, producing the widest plume, although still, steep gradients are present in the plume.

\section{SUMMARY AND CONCLUDING REMARKS}

The two-staged TIHTUS can be operated in purely arc heated, purely inductively heated, or combined operation. It can be continuously operated with a power of $50 \mathrm{~kW}$.

Pitot pressure and plasma velocity were measured in a radially resolved manner at an axial downstream position of $x=200 \mathrm{~mm}$ from the exhaust plane. Total pressure maximum is at $85 \mathrm{~Pa}$, while maximum velocity is measured at $9,224 \mathrm{~m} / \mathrm{s}$ for T $50|00-300| 0$ and $10,350 \mathrm{~m} / \mathrm{s}$ for T $25|25-200| 100$. The pressure profile exhibits mostly the "M"-shaped behavior indicating that the plume is a turbulent free jet. Velocity profile shape is dependent on gas-flow staging. The maxima move to eccentric positions with increasing swirl gas injected.

From both, pressure and velocity pofiles, it is shown that power coupling of the second stage takes place in the typical off-axis position close to the discharge coil.

A method was introduced to determine radially resolved temperature from the total pressure and velocity profiles. The plasma temperature maxima measured are $8689 \mathrm{~K}$ and $>8000 \mathrm{~K}$ for T $50|00-300| 0$ and T $25|25-200| 100$, respectively. Large temperature and velocity are detected at T 25|25-200|100, making them the favoured operating condition for high plasma velocity while for high total pressure, T 20|30-300|0 provides the highest integral Pitot pressure.

The Pitot pressure measurements are compared to thrust measurement in [4]. For completion of the presented work, temperature needs to be measured directly, possibly by optical methods or by crossed electric single probes. 


\section{ACKNOWLEDGMENTS}

The authors would like to thank Dr. Thomas Stöckle for initiating the idea of temperature deduction, Dr. Stefan Löhle for fruitful discussions and their students Melanie Lücke and Markus Mayer for their contribution to the presented experiments.

\section{REFERENCES}

1. Bonneville, J. M. 1963. High-frequency supersonic heating of hydrogen for propulsion. AIAA Electric Propulsion Conference. Colorado Springs, CO.

2. Wallner, L.E., and J. Czika. 1965. Arc-jet thrustor for space propulsion. Tech. Report NASA TN D-2868. NASA, Lewis Research Center, Cleveland, USA.

3. Boehrk, H., and M. Auweter-Kurtz. 2006. Preliminary results of TIHTUS operation. AIAA Paper No. 2006-5158.

4. Böhrk, H., and M. Auweter-Kurtz. 2008 (submitted). Thrust measurement of the 2008. Two-stage electric thruster TIHTUS by a baffle plate. J. Propul. Power. (Also: 2007. TIHTUS thrust measurement with a baffle plate. AIAA Paper No.20075297.)

5. Böhrk, H., S. Laure, and M. Auweter-Kurtz. 2004. Feasibility study of application of ATTILA to in-space propulsion for piloted missons. Astronautical Conference (International). IAC-04-S.4.03. Vancouver, Kanada.

6. Böhrk, H., T. Schmidt, and M. Auweter-Kurtz. 2007. Flexible piloted Mars missions using the TIHTUS engine. Aerospace Sci. Technol. 11(2-3):211-21.

7. Eckert, H. U. 1974. The induction arc: A state-of-the-art review. J. High Temp. Sci. 6:99-134.

8. Liebeskind, J. G., R. K. Hanson, and M. A. Cappelli. 1993. Laser-induced fluorescence diagnostic for temperature and velocity measurements in a hydrogen arcjet plume. Appl. Opt. 32(30):6117-27.

9. Scott, C. D. 1993. Survey of measurements of flow properties in arcjets. J. Thermophys. Heat Transfer 7(1):9-24.

10. Scheubert, P., U. Fantz, P. Awakovicz, and H. Paulin. 2001. Experimental and theoretical characterization of an inductively coupled plasma source. J. Appl. Phys. 90(2):587-98.

11. Habiger, S. 1994. Elektrostatische Sonden und Fabry-Perot Interferometrie zur Untersuchung von lichtbogenbeheizten Plasmen für Triebwerksanwendungen und Wiedereintrittssimulation. Ph.D. Thesis. Universität Stuttgart, Fak. Luft- und Raumfahrttechnik.

12. Löhle, S. 2006. Experimentelle Untersuchung von Wiedereintrittsplasmen mit Hilfe laserinduzierter Fluoreszenzmessungen. Ph.D. Thesis. Universität Stuttgart, Fak. Luft- und Raumfahrttechnik.

13. Poissant, G., and M. Dudeck. 1985. Velocity profiles in a rarefied argon plasma stream by crossed electrostatic probes. J. Appl. Phys. 58(5):1772-79. 
14. Malliaris, A.C. 1966. Induction velometry in an ionized gas. J. Appl. Phys. $37(11): 4159-68$.

15. Liepmann, H.W., and A. Roshko. 1960. Elements of gasdynamics. 3rd ed. N.Y.London: John Wiley and Sons, Inc.

16. Krause, S. 1977. Pitotsonden in magnetofluiddynamischen Strömungen. Ph.D. Thesis. Universität Stuttgart, Fak. Luft- und Raumfahrttechnik.

17. Hoell, J. M., J. Burlock, and O. Jarrett, Jr. 1971. Velocity and thrust measurements in a quasi-steady magnetoplasmadynamic thruster. AIAA J. 9(10):1969-74.

18. Laure, S. 1998. Experimentelle Simulation der Staupunktströmung wiedereintretender Raumflugkörper und deren Charakterisierung mittels mechanischer Sonden. Ph.D. Thesis. Universität Stuttgart, Fak. Luft- und Raumfahrttechnik.

19. Hiers, R., and D. Pruitt. 2001. Determination of thrust from pitot pressure measurements. AIAA Paper No. 2001-3314.

20. Abramovich, G. N. 1963. Theory of turbulent jets. MIT Press.

21. Laure, S., M. Auweter-Kurtz, S. Fasoulas, H. Habiger, H., and A. Schönemann. 1994. Experimentelle Simulation einer hochenthalpen Luftströmung im Plasmawindkanal. Jahrbuch der deutschen Gesellschaft für Luft- und Raumfahrt 3. DGLR, Erlangen.

22. Patch, R. W. 1971. Thermodynamic properties and theoretical rocket performance of hydrogen to $100,000 \mathrm{~K}$ and $1.01325 \times 10^{8} \mathrm{~N} / \mathrm{m}^{2}$. Tech. Report NASA SP-3069. NASA, Lewis Research Center, Cleveland. 